\title{
Sessile serrated lesions: Searching for the true prevalence
}

\section{다 (i) $(-)$}

\author{
Authors \\ Charles J. Kahi, Douglas K. Rex
}

Institution

Indiana University School of Medicine, Indianapolis, Indiana, United States

\section{Bibliography}

Endosc Int Open 2021; 09: E635-E636

DOI 10.1055/a-1373-4825

ISSN 2364-3722

(C) 2021. The Author(s).

This is an open access article published by Thieme under the terms of the Creative Commons Attribution-NonDerivative-NonCommercial License, permitting copying

\author{
and reproduction so long as the original work is given appropriate credit. Contents \\ may not be used for commercial purposes, or adapted, remixed, transformed or \\ built upon. (https://creativecommons.org/licenses/by-nc-nd/4.0/) \\ Georg Thieme Verlag KG, Rüdigerstraße 14, \\ 70469 Stuttgart, Germany \\ Corresponding author \\ Charles J. Kahi, Professor of Medicine, Indiana University \\ School of Medicine, 702 Rotary Circle Suite 225, Indianapolis, \\ IN 46202, United States \\ ckahi2@iu.edu
}

Reality is merely an illusion, albeit a very persistent one - Albert Einstein

The serrated class of colorectal polyps includes hyperplastic polyps (HP), sessile serrated lesions (SSL), and traditional serrated adenomas (TSA). Among these, SSL are the most screeningrelevant, because they are considered the main precursor lesion in the serrated colorectal cancer (CRC) pathway, and are much more prevalent than TSAs [1]. The growing recognition of the importance of SSLs has been accompanied by numerous studies reporting detection rates at colonoscopy. Inevitably, systematic reviews and meta-analyses have followed, reporting aggregates of available SSL detection studies. It is important here to distinguish between aspirational detection rates, which are more reflective of the true population-based SSL prevalence, and the more commonly reported overall SSL detection rates among groups of endoscopists.

In this issue of EIO, Zheng and Wong selected 17 studies totaling 129,000 average-risk patients, and reported serrated lesion, SSL, and TSA detection rates of $19 \%, 2.5 \%$, and $0.3 \%$, respectively. Rates of SSL detection were similar in men and women, but varied significantly based on race (26\% in Whites vs. $15 \%$ in Asians) and geographic setting. Another recent systematic review [2] by Meester et al. included 74 colonoscopy studies (unrestricted by indication) and found an overall SSL prevalence of $4.6 \%$, which increased to $9 \%$ when only highquality colonoscopies were considered. Similar to the current analysis, they found no differences by sex (nor age), and substantial variability according to world region.

The effort to determine the true prevalence of SSL is important, because setting aspirational detection targets for endoscopists ultimately improves the effectiveness of CRC prevention with high-quality colonoscopy. However, using meta-anal- ysis of observational studies to determine SSL prevalence has limited utility, because this ultimately reflects the mean of different centers, and each report or study in the analysis (with rare exception) reflects the average performance of multiple endoscopists with widely variable detection rates. Several studies have in fact shown serrated lesion detection variability that far exceeds that for adenomas among the same group of endoscopists [3-5]. Determining true prevalence has to start with studies reporting the highest detection levels, and our group [6] and others [7] have done such an exercise: in patients who had undergone colonoscopy by endoscopists with high adenoma and SSL detection rates combined with histological review by expert gastrointestinal pathologists, SSL prevalence was $8 \%$ to $9 \%$.

However, determining the true prevalence of SSLs is a more complex proposition than for adenomas, and not simply a matter of reporting detection rates at colonoscopy. In the case of adenomas, we have a clearer understanding, because numerous studies have reported individual rates, thus allowing discernment of the highest detection levels, and because the histopathological distinction between adenomas and serrated class lesions is straightforward and reliable. For SSLs, understanding true prevalence is complicated by several factors, in addition to the paucity of studies reporting individual and aspirational SSL detection rates. These include the lack of a validated definition for SSL, the existence of multiple histological definitions for SSL vs HP, and histological criteria based on the presence and number of distorted gland crypts, which are inherently subjective and prone to interobserver variability [8], including among expert pathologists. In addition, studies have shown a trend of rising rates of SSL diagnoses, which suggests 
increased pathologist awareness or bias to diagnose SSL, especially for larger lesions and those located in the right colon [1].

The bottom line is that the true prevalence of SSL is a moving target, not just dependent on local endoscopic and pathology practice and bias, but also on geography and race/ethnicity. This may frustrate those among us who seek a clarity comparable to that of the adenoma detection rate (ADR), and its inverse association with post-colonoscopy CRC (PCCRC) risk [9, $10]$. Where do we go from here? Perhaps, for now, the best approach is decentralization: instead of one universal SSL metric, we should study detection rates of endoscopists within institutions, and then use the highest detection as the local aspirational target. This approach is dissimilar to ADR, but could allow practices to catch up with the evolving endoscopic and histopathological definitions of SSL, and compare local rates to the aspirational ones reported by expert groups.

\section{Competing interests}

The authors declare that they have no conflict of interest.

\section{References}

[1] Crockett SD, Nagtegaal ID. Terminology, molecular features, epidemiology, and management of serrated colorectal neoplasia. Gastroenterology 2019; 157: 949-966 e4

[2] Meester RGS, van Herk M, Lansdorp-Vogelaar I et al. Prevalence and clinical features of sessile serrated polyps: a systematic review. Gastroenterology 2020; 159: 105-118 e25

[3] Hetzel JT, Huang CS, Coukos JA et al. Variation in the detection of serrated polyps in an average risk colorectal cancer screening cohort. Am J Gastroenterol 2010; 105: 2656-2664

[4] Kahi C], Hewett DG, Norton DL et al. Prevalence and variable detection of proximal colon serrated polyps during screening colonoscopy. Clin Gastroenterol Hepatol 2011; 9: 42-46

[5] de Wijkerslooth TR, Stoop EM, Bossuyt PM et al. Differences in proximal serrated polyp detection among endoscopists are associated with variability in withdrawal time. Gastrointest Endosc 2013; 77: 617-623

[6] Abdeljawad K, Vemulapalli KC, Kahi C] et al. Sessile serrated polyp prevalence determined by a colonoscopist with a high lesion detection rate and an experienced pathologist. Gastrointest Endosc 2015; 81: 517-524

[7] IJspeert JGE, de Wit K, van der Vlugt $\mathrm{M}$ et al. Prevalence, distribution and risk of sessile serrated adenomas/polyps at a center with a high adenoma detection rate and experienced pathologists. Endoscopy 2016; 48: 740-746

[8] WHO Classification of Tumours: digestive system tumours. 5th edition. Lyon: International Agency for Research on Cancer; 2019

[9] Corley DA, Levin TR, Doubeni CA. Adenoma detection rate and risk of colorectal cancer and death. N Engl J Med 2014; 370: 2541

[10] Kaminski MF, Regula J, Kraszewska E et al. Quality indicators for colonoscopy and the risk of interval cancer. N Engl J Med 2010; 362: 1795-803 